\title{
An Active Microchannel Neural Interface with Artifact Reduction
}

\author{
Maryam Habibollahi ${ }^{1}$, Farnaz Fahimi Hanzaee ${ }^{1}$, Dai Jiang ${ }^{1}$, Henry Lancashire ${ }^{2}$, and Andreas Demosthenous ${ }^{1}$ \\ ${ }^{1}$ Department of Electronic and Electrical Engineering, University College London, Torrington Place, London WC1E 7JE, UK \\ ${ }^{2}$ Department of Medical Physics and Biomedical Engineering, University College London, London, WC1E 6BT, UK \\ e-mail: maryam.habibollahi.15@ucl.ac.uk; a.demosthenous@ucl.ac.uk
}

\begin{abstract}
High-density neural electrodes in microchannel interfaces require in-situ amplification of the neural signals and rejection of high-voltage stimulus pulses leaking to the channel in order to adequately detect neural signals in the presence of concurrent stimulation. This paper presents the design of an active microchannel neural interface in 0.18 $\mu \mathrm{m}$ CMOS employing neural recording and stimulation. To reduce stimulus artifacts, a novel method is proposed that disconnects the recording module during concurrent channel stimulation and automatically applies detection and reduction of stimulus artifacts from adjacent channels using a tunable filter. Simulations show that the method provides at least $54 \mathrm{~dB}$ artifact attenuation.
\end{abstract}

Index Terms - Neural interface, biomedical electrodes, neural recording, microchannels, high-voltage stimulation, artifact detection

\section{INTRODUCTION}

Regenerative neural interfaces selectively interface subpopulations of neurons using separate electrodes, while offering a trade-off between encouraging axonal growth and selectivity [1]. Microchannel neural interfaces (MNI) are regenerative interfaces which maximize the amplitude of recorded action potentials by confining the extracellular space surrounding the axon [1]. As shown in Figure 1a and Figure $1 \mathrm{~b}$, MNIs comprise parallel tubes (channels) formed of a biocompatible insulator such as silicone [2] or polyimide [1], containing electrode contacts. Microchannel lengths typically range from $2 \mathrm{~mm}$ to $5 \mathrm{~mm}$ for nerve regeneration; this allows neural recording independent of the location of the nodes of Ranvier in the MNI [1]. A channel width of $100 \mu \mathrm{m}$ to $200 \mu \mathrm{m}$ achieves a selective interface with a large extracellular resistance, while limiting channel blockage by fibrotic tissue [1].

Channel density in passive MNIs is limited by the need for interconnects for each electrode [3]. Furthermore, the use of tripolar electrodes, a method of artifact reduction, requires additional interconnects per channel, increasing design complexity and the risk of device failure. To overcome such challenges, a stimulator-recording system with miniaturized on-chip connections was reported, [3] with electrodes on the surface of an application specific integrated circuit (ASIC) to multiplex all the corresponding connections. As a result, fewer and shorter interconnects are required to access the channels.

This work was supported in part by the European Commission under H2020- EU.1.2.2. - FET Proactive (Agreement ID: 824071; project

NeuHeart; http://www.neuhearth2020.eu/) and in part by the

Engineering and Physical Sciences Research Council (EPSRC).
Further scaling could be achieved by stacking several parallel ASICs to create a 3-dimensional microchannel array, as shown in Figure 1c.

Adequate recording of neural signals requires a sufficiently high input range and signal-to-noise ratio to prevent signal distortion, as well as a large enough common-mode rejection ratio, which is often limited by channel interferences and mismatch in the electrode impedances. In-situ amplifiers have been developed to address this issue by reducing the capacitive coupling of the connective tracks. The resulting active electrodes can convert the electrode sites to low-impedance nodes, limiting their sensitivity to crosstalk and other artifacts [4].

A challenge is imposed, however, for concurrent stimulation and recording with an MNI. Due to the large electrode impedances and the required stimulus currents reported on microchannel devices, very high stimulus compliance voltages $(40 \mathrm{~V})$ can appear at the inputs of the recording front-end during concurrent channel stimulation. To address this, a method is proposed in this paper that minimizes artifacts during recording. This can be addressed by temporarily disconnecting the recording unit to block the stimulus artifact. Moreover, in-band artifacts can be detected at the recording input from the adjacent channels' stimulus pulses, which can be automatically detected to enable adjustment of the filter characteristics.

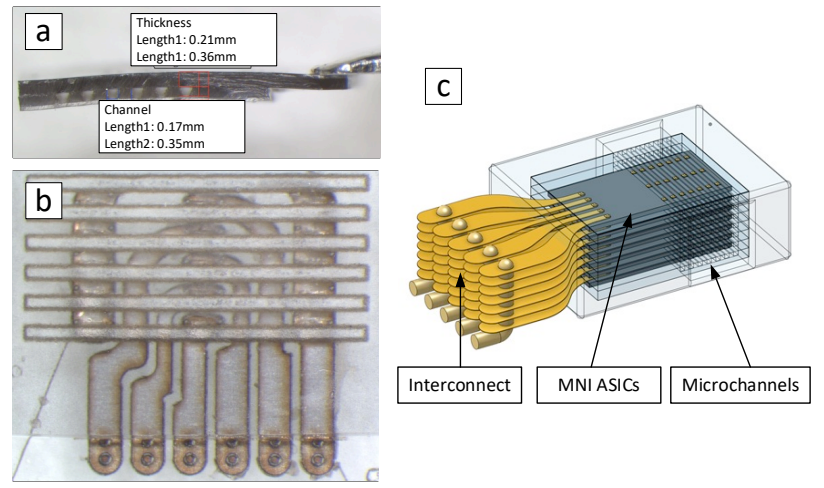

Figure 1. A stacked MNI device showing the (a) layer thickness, (b) channel surface area, and (c) multi-layer view [3].

The paper is organized as follows. Section II reviews the conventional methods used for artifact reduction and introduces the proposed novel technique. Section III provides an overview of the active MNI concept and the circuitry for artifact detection and reduction during recording. Section IV presents the simulation results. Comparison with other designs is detailed in Section V, and concluding remarks are presented in Section VI. 


\section{ARtifact Reduction Methods}

\section{A. Conventional Methods}

Commonly used techniques that mitigate the effects of stimulus artifacts can be divided into three categories: prevention, front-end immunity, and back-end processing [5]. Preventive methods such as charge balancing ease the requirement on the front-end, though fail to remove artifacts sufficiently, leading to amplifier saturation and slow amplifier recovery. Alternative techniques that improve the front-end immunity aim to prevent saturation to maintain a linear response [6], or achieve rapid recovery for reduced data loss [7]. Back-end cancellation methods are often applied to digitized signals to reconstruct the neural data or subtract artifacts via adaptive filtering [5], achieving reliable artifact removal at the cost of area and complexity [8].

\section{B. Proposed Method}

This work proposes an automatic and simple design for artifact reduction, combining two methods to increase front-end resilience to high-voltage artifacts. Namely, signal blanking, which protects the recording amplifier inputs, easing the requirements for a high dynamic range and allowing a lowvoltage implementation of the recording front-end, and pole shifting, which reduces the effects of adjacent channel pulses and minimizes data loss by adjusting the low-pass cut-off frequency of the amplifier during adjacent channel stimulation.

An additional means of further reducing the artifacts is via the application of chopped stimulus pulses, which consist of high-frequency current packets within each pulse with small intervals between pulses. Compared to the conventional lumped pulse technique, which applies current pulses hundreds of microseconds wide, the $\mu$ s-wide current packets of the chopped pulses result in high frequency outputs due to the resistive component of the electrode-electrolyte interface, while the envelope generated by the capacitive components of the interface can appear at much lower amplitudes [9]. Consequently, the artifacts due to chopped pulses can be filtered more effectively. The pixel-based structure of the recording and stimulation circuitry for each channel in the MNI allows for individual and customized control of each electrode.

\section{System Design}

Figure 2 shows an overall block diagram of the active MNI ASIC, composed of seven tripolar electrode channels, such as those shown in Figure 1b, alongside a global control and biasing unit. All channels include a localized recording module that operates the artifact reduction circuits, and a stimulation module, the parameters of which are directly set by the local digital control unit. A global $2^{\text {nd }}$-order low-pass filter is included in each chip to provide a sharper roll-off, thus attenuating the artifacts more distinctly.

The primary goal of the system is to apply closed-loop stimulation by recording the neural signals generated via the stimulus pulses. To achieve this, a bandwidth of $300 \mathrm{~Hz}$ to 5 $\mathrm{kHz}$ [3] is selected for detecting action potentials with amplitudes ranging from $20 \mu \mathrm{V}$ [1] to $520 \mu \mathrm{V}$ [10] under nominal recording conditions, during which no stimulus artefacts from adjacent channels are detected.
A pole shifting mechanism is implemented to limit the effects of crosstalk during concurrent stimulation and recording by constricting the recording bandwidth of the amplifier [11]. This function is automatically enabled upon the detection of higherthan-expected input signals via a differential comparator, as discussed in the following sub-sections.

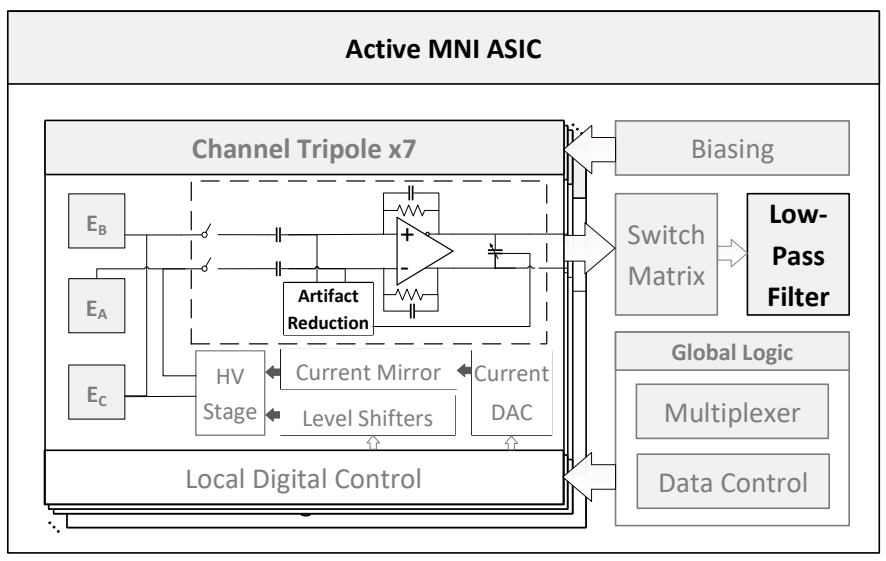

Figure 2. Active MNI system block diagram.

\section{A. Neural Recording}

The fully differential low-noise amplifier (LNA) illustrated in Figure 3a was implemented with a capacitive feedback network to remove all DC offsets from the inputs and define a mid-band gain of $C_{i n} / C_{f}=40 \mathrm{~dB}$. A feedback resistor $R_{f}$ is placed in parallel with the capacitor $C_{f}$ to bias the input terminals and define the high-pass corner. The load capacitor $C_{L}$ connected across the outputs selects a tunable low-pass corner.

Two high-voltage (HV) switches, $\mathrm{M}_{\mathrm{H} 0}$ and $\mathrm{M}_{\mathrm{H} 1}$, are included at the amplifier inputs to provide blanking of the stimulus pulses in the same channel, which can exceed the safe limits specified for the low-voltage (LV) transistors. The use of protective HV switches allows the safe operation of the HV stimulator unit alongside the LV recording circuitry, which consumes less power and a smaller chip area.

A telescopic cascode operational transconductance amplifier (OTA) with common-mode feedback was selected to detect neural signals in the specified range as shown in Figure $3 \mathrm{~b}$. The dimensions of input transistors $M_{1}$ and $M_{2}$ were carefully selected to minimize the flicker noise introduced by operating in the weak inversion region. Conversely, the $g_{m} / I_{d}$ ratio of the load transistors $M_{7}$ and $M_{8}$ was minimized to operate in strong inversion for a low thermal noise.

The pseudo-resistor $R_{f}$ was implemented using a parallelNMOS architecture. This structure is selected to optimize the control and performance of the feedback resistor under the effects of PVT variations with minimal noise and power consumption. 


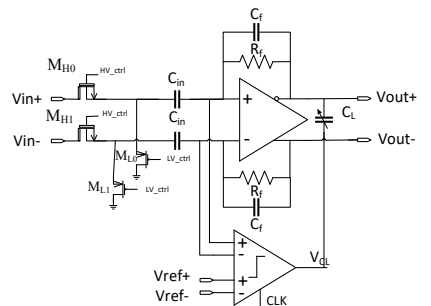

(a)

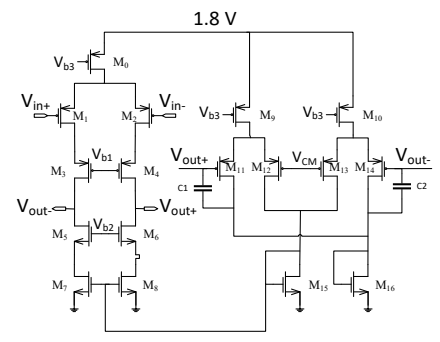

(b)
Figure 3. LNA schematics showing (a) amplifier with blanking and artifact detection, and (b) OTA with common-mode feedback.

\section{B. Artifact Detection}

In order to accurately record neural activity without saturation by artifacts generated from adjacent channels' stimulus pulses, the recording amplifier provides a variable bandwidth to block the high-amplitude stimulus pulses. The application of pole shifting provides front-end immunity to the amplifier against stimulus artifacts, thus reducing data loss without the need for a high dynamic range [5]. A similar technique more often described as 'soft switching' takes advantage of the adjustability of passive components such as pseudo-resistors and variable capacitors in controlling the bandwidth, limiting the effects of switching artifacts such as leakage and charge injection [11]. This also supports rapid recovery for the front-end and does not require electrode discharge for every event of adjacent channel stimulation, as is the case following amplifier disconnection during blanking, which is necessary to protect the LV recording unit [7].

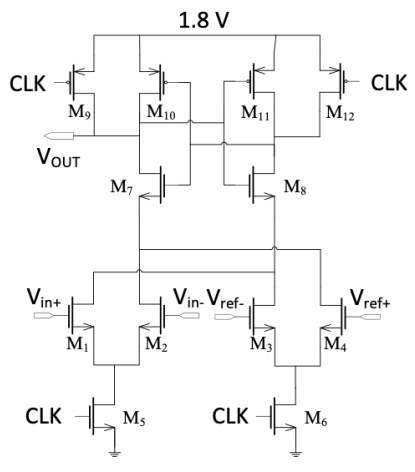

(a)

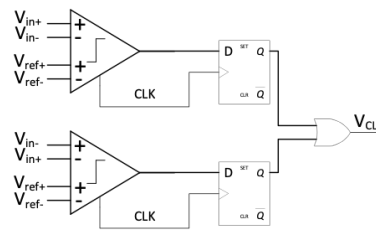

(b)

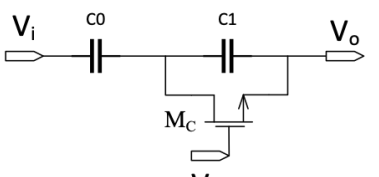

(c)
Figure 4. Schematic diagram of (a) fully differential comparator, (b) logic output stage, and (c) variable load capacitor.

Timely detection of artifacts is achieved via the fully differential comparator shown in Figure 3a and Figure 4a. A latched architecture is implemented to achieve a high speed without compromising power or area, while the differential sensing inputs account for the voltage excursions expected in the recorded neural signals. In order to enable pole shifting upon the detection of high input signals at either input, thus accounting for both anodic-first and cathodic-first stimulus pulses, two such comparators are placed in each channel as shown Figure 4b, with a control signal extracted using D flipflops and an OR gate.

A schematic of the variable load capacitor $\left(C_{L}\right)$ is presented in Figure 4c, where the comparator output determines the state of transistor switch, $M_{C}$, via its gate control signal, $V_{C L}$. During pole shifting, the switch bypasses the capacitor $C_{1}$ and provides a load capacitance equal to $C_{0}$, while during nominal operation, the switch is off, and the load capacitor is a series combination of $C_{0}$ and $C_{1}$.

\section{Simulation Results}

A microchannel neural interface using the proposed circuits was designed and simulated in a $0.18 \mu \mathrm{m}$ HV CMOS technology. The performance of the recording unit is presented below.

\section{A. Recording Amplifier}

The capacitively-coupled LNA is designed to provide a total gain of $40 \mathrm{~dB}$ via the input and feedback capacitors set at $20 \mathrm{pF}$ and $200 \mathrm{fF}$, respectively. To obtain the nominal bandwidth for action potentials $(300 \mathrm{~Hz}$ to $5 \mathrm{kHz}$ ), the feedback resistor is tuned to $2.5 \mathrm{M} \Omega$, and the series load capacitors to $2.6 \mathrm{fF}$ across the outputs of the amplifier. The OTA has an input-referred noise of $5.2 \mu \mathrm{V}_{\mathrm{rms}}$. This proves a sufficiently low noise floor, as the recorded action potential signal magnitudes in microchannels range from $20 \mu \mathrm{V}$ to around $520 \mu \mathrm{V}$.

The high-voltage blanking switches at the amplifier inputs block HV signals by providing an off-resistance of $5.5 \mathrm{M} \Omega$ during same-channel stimulation and present an on-resistance of up to $500 \Omega$ during recording, which is at least two orders of magnitude smaller than the expected electrode impedance [1]. Monte Carlo analysis was carried out to measure the variation in the value of the pseudo-resistor, $\mathrm{R}_{\mathrm{f}}$. The average resistance was measured within $8.5 \%$ of the nominal value, with a standard deviation of $1.8 \%$. This corresponds to a maximum difference of $0.21 \mathrm{G} \Omega$, which results in up to $23 \mathrm{~Hz}$ of variation in the frequency domain.

\section{B. Bandwidth Adjustment}

Figure 5a shows the frequency response of the recording amplifier over the nominal action potential range and the modified range upon the application of artifact reduction that shifts the low-pass cut-off frequency of the filter to $1 \mathrm{kHz}$, which is below the typical stimulus pulse frequency range.

Transient analyses of the differential comparator demonstrated the pole shifting functionality over inputs above and below the comparator threshold voltage. A maximum delay of $20 \mathrm{~ns}$ was measured on the rising edge of the comparator for a clock period of $10 \mu \mathrm{s}$, resulting in a minimum slew rate of $88.6 \mathrm{~V} / \mu \mathrm{s}$. This proves a sufficiently fast response for artifacts with frequencies as high as $20 \mathrm{kHz}$, which are used to block nerve conduction [12]. The reduction capability of the pole shifting module was tested by measuring the LNA outputs of a composite input signal that consists of stimulation artifacts 
generated as $30 \mathrm{mV}$ pulses with the commonly-applied pulse width of $0.2 \mathrm{~ms}$ [13], and recording spikes $500 \mu \mathrm{V}$ in amplitude applied at a $1 \mathrm{kHz}$ frequency, resulting in a $-36 \mathrm{~dB}$ artifact-tosignal ratio at the inputs. Figure $5 \mathrm{~b}$ illustrates the effect of pole shifting on the LNA output signals in the time domain. This method achieves an attenuation ratio of $54 \mathrm{~dB}$ with chopped stimulus artifacts.

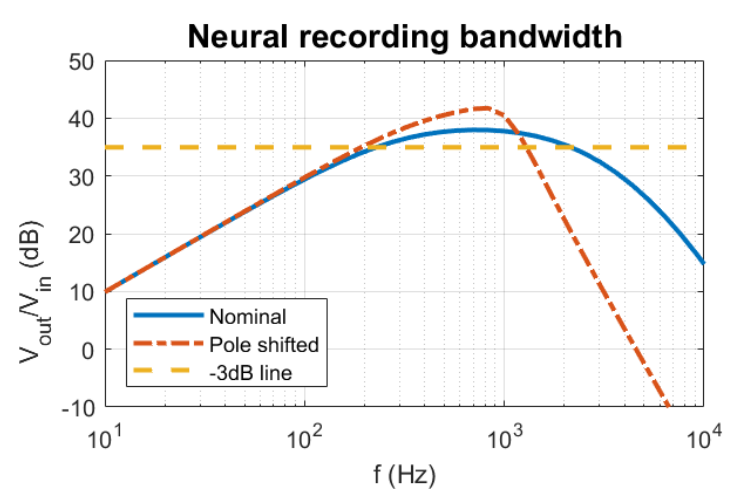

(a)

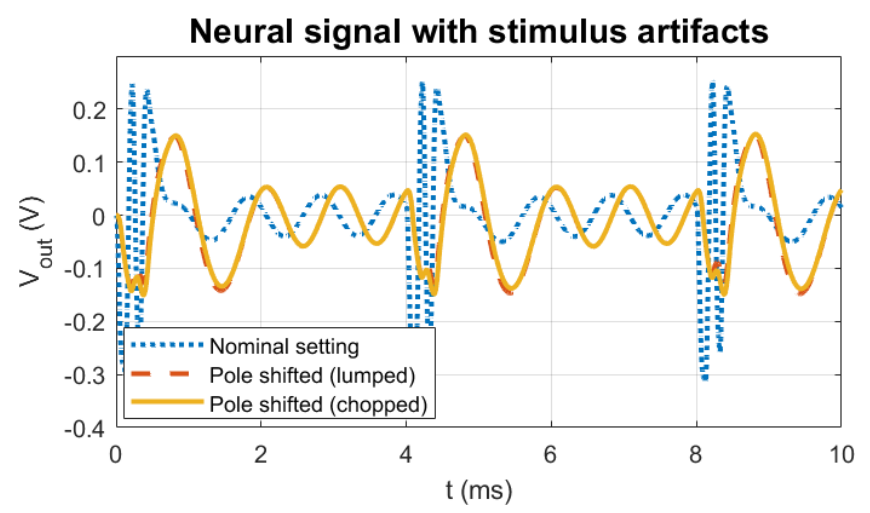

(b)

Figure 5. Simulation results of (a) LNA bandwidth adjustment and (b) Artifact attenuation with chopped and lumped pulses.

\section{DISCUSSION AND CONCLUSION}

This design demonstrates an on-board recording and stimulation system for microchannel applications, capable of detecting artifacts generated due to the high-voltage stimulus pulses that exceed the expected magnitude range of the action potential signals in each channel. The combination of the capacitively-coupled LNA and the comparators occupies an area of $0.23 \mathrm{~mm}^{2}$ and consumes up to $3.89 \mu \mathrm{W}$ of power during artifact reduction. The design achieves an acceptable trade-off between the simulated power and noise. It has an input-referred noise of $6.3 \mu \mathrm{V}_{\text {rms }}$ and a noise efficiency factor of 5.13 over the recording frequency range of $300 \mathrm{~Hz}$ to $5 \mathrm{kHz}$. Future work will include improving the signal-to-noise ratio of the LNA by optimizing the low-pass filter that acts to reduce artifacts.
An active MNI system for the stimulation and recording of nerves enclosed in microchannels has been presented and simulated in $0.18 \mu \mathrm{m}$ CMOS. The concept of artifact detection via the fully differential comparator and its reduction using the pole shifting technique has been explained and verified using Cadence Spectre. An attenuation ratio of at least $54 \mathrm{~dB}$ has been demonstrated in reducing stimulus artifacts.

\section{REFERENCES}

[1] J. J. FitzGerald, N. Lago, S. Benmerah, J. Serra, C. P. Watling, R. E. Cameron, E. Tarte, S. P. Lacour, S. B. McMahon and J. W. Fawcett, "A regenerative microchannel neural interface for recording from and stimulating peripheral axons in vivo," J. Neural Eng., vol. 9, no. 1, p. 016010, Feb. 2012.

[2] H. T. Lancashire, A. Vanhoestenberghe, C. J. Pendegrass, Y. A. Ajam, E. Magee, N. Donaldson and G. W. Blunn, "Microchannel neural interface manufacture by stacking silicone and metal foil laminae," Journal of Neural Engineering, vol. 13, no. 3, pp. 1-9, 16.

[3] H. Lancashire, D. Jiang, A. Demosthenous and N. Donaldson, "An ASIC for Recording and Stimulation in Stacked Microchannel Neural Interfaces," IEEE Trans. Biomed. Circuits Syst., vol. 13, no. 2, pp. 259270, 2019.

[4] C. M. Lopez, A. Andrei, S. Mitra, M. Welkenhuysen, W. Eberle, C. Bartic, R. Puers, R. F. Yazicioglu and G. G. Gielen, "An implantable 455-active-electrode 52-channel CMOS neural probe," IEEE Journal of Solid-State Circuits, vol. 49, no. 1, pp. 248-261, 2014.

[5] A. Zhou, B. C. Johnson and R. Muller, "Toward true closed-loop neuromodulation: artifact-free recording during stimulation," Current Opinion in Neurobiology, vol. 50, pp. 119-127, 2018.

[6] P. Hottowy, A. Skoczeń, D. E. Gunning, S. Kachiguine, K. Mathieson, A. Sher, P. Wiącek, A. M. Litke and W. Dạbrowski, "Properties and application of a multichannel integrated circuit for low-artifact, patterned electrical stimulation of neural tissue," Journal of Neural Engineering, vol. 9, no. 6, 2012.

[7] V. Viswam, Y. Chen, A. Shadmani, J. Dragas, R. Bounik, R. Milos, J. Muller and A. Hierlemann, "2048 Action Potential Recording Channels With $2.4 \mathrm{uVrms}$ Noise and Stimulation Artifact Suppression," in IEEE Biomed. Circuits Syst. Conf., 2016.

[8] J. P. Uehlin, W. A. Smith, V. R. Pamula, E. P. Pepin, S. Perlmutter, V. Sathe and J. C. Rudell, "A Single-Chip Bidirectional Neural Interface With High-Voltage Stimulation and Adaptive Artifact Cancellation in Standard CMOS," IEEE J. Solid-State Circuits, pp. 1-13, 2020.

[9] D. Jiang and A. Demosthenous, "A Multichannel High-Frequency Power-Isolated Neural Stimulator with Crosstalk Reduction," IEEE Trans. Biomed. Circuits Syst., vol. 12, no. 4, pp. 940-953, 2018.

[10] A. Srinivasan, M. Tahilramani, J. T. Bentley, R. K. Gore, D. C. Millard, V. J. Mukhatyar, A. Joseph, A. S. Haque, G. B. Stanley, A. W. English and R. V. Bellamkonda, "Microchannel-based regenerative scaffold for chronic peripheral nerve interfacing in amputees," Biomaterials, vol. 41, pp. 151-165, Feb. 2015.

[11] A. Shadmani, V. Viswam, Y. Chen, R. Bounik, J. Dragas, M. Radivojevic, S. Geissler, S. Sitnikov, J. Muller and A. Hierlemann, "Stimulation and Artifact-suppression Techniques for in-vitro Highdensity Microelectrode Array Systems," IEEE Trans. Biomed. Eng., vol. 66, no. 9, pp. 2481-2490, 2019.

[12] D. J. Chew, L. Zhu, E. Delivopoulos, I. R. Minev, K. M. Musick, C. A. Mosse, M. Craggs, N. Donaldson, S. P. Lacour, S. B. McMahon and J. W. Fawcett, "A Microchannel Neuroprosthesis for Bladder Control After Spinal Cord Injury in Rat," Neuroprosthetics, vol. 5, no. 210, 2013.

[13] H. Yuan and S. D. Silberstein, "Vagus Nerve and Vagus Nerve Stimulation, a Comprehensive Review: Part III," American Headache Society, vol. 56, no. 3, pp. 479-490, 2015. 